\title{
Study on Cone-defects during the Pattern Fabrication Process with Silicon Nitride
}

\author{
Takuya Hagiwara ${ }^{1}$, Kentaro Saito ${ }^{1}$, Hiraku Chakihara ${ }^{1}$, Shuji Matsuo ${ }^{2}$, \\ Masao Inoue ${ }^{1}$, Seiji Muranaka ${ }^{1}$, Yuki Ota $^{2}$ and Masazumi Matsuura ${ }^{1}$ \\ ${ }^{1}$ Renesas Electronics Corporation, \\ 751 Horiguchi, Hitachinaka, Ibaraki 312-8504, Japan \\ ${ }^{2}$ Renesas Semiconductor Manufacturing Co., Ltd., \\ 751 Horiguchi, Hitachinaka, Ibaraki 312-8504, Japan
}

\begin{abstract}
When resist patterns are formed directly on silicon nitride ( $\mathrm{SiN}$ ), it is common for cone defects to arise after the SiN dry-etching process. When subsequent layers are deposited, the layers pile up around a cone defect like a side wall, causing it to expand. These expanded cone defects can cause short circuits in the pattern. To improve yields, therefore, it is vital to understand the factors that lead to the occurrence of cone defects and those which suppress their generation. As a result of our investigations, we were able to determine that these cone defects are caused by metal impurities (especially, iron atoms) in the developer. These become trapped in the resist residue. The complex that is formed by the polymer in the resist residue and the metal then acts as an etching mask. We also determined that trap sites, where the metal can settle, exist on the $\mathrm{SiN}$ surface and the metal atoms that thus accumulate on the SiN surface act as an etching mask. Given these results, the number of cone defects can be reduced by suppressing this resist residue generation through the use of high-contrast resists and reducing the metallic impurities in both the developer and chemicals discharged onto SiN.
\end{abstract}

Keywords: cone defect, silicon nitride, resist residue, metallic impurities, iron atom, developer, swelling, polar group

\section{Introduction}

Silicon nitride ( $\mathrm{SiN}$ ) plays an important role in the manufacture of semiconductor devices. Given its high oxidation resistance (compared to silicon) and excellent ability to prevent sodium $(\mathrm{Na})$ contamination and moisture penetration, $\mathrm{SiN}$ is frequently used as a selective oxidation mask and passivation film. It is also widely used to fabricate dynamic random access memory (DRAM) capacitors and as an insulating film for metal nitride oxide semiconductor (MNOS) nonvolatile memory. However, from the viewpoint of lithographic patterning, a number of issues arise with the application of SiN. For example, when a resist pattern is formed on $\mathrm{SiN}$, an acid deactivation reaction takes place between the resist and the SiN interface. This results in the formation of a pattern footing and resist residue generation [1-4]. Another issue is the occurrence of cone defects after the dry-etching process of SiN, when the resist pattern is fabricated directly on the SiN. These cone defects are generally small, and thus are not regarded as being critical defects. If another layer is applied after the generation of a cone defect, however, these layers pile up around the cone defect like a side wall, causing the cone defect to expand. Cone defects that have expanded in this way can easily cause short circuits. Therefore, to improve semiconductor yields, it is vital to suppress the generation of cone defects. In this paper, the results of our investigations into the cause of cone defect generation are discussed in 
detail. Moreover, proposals for suppressing the occurrence of these cone defects are also presented.

\section{Experimental}

\subsection{Resist patterning conditions}

To investigate the causes of cone defect generation, $\mathrm{SiN}$ and silicon dioxide $\left(\mathrm{SiO}_{2}\right)$ substrate surfaces were used. $90 \mathrm{~nm}$ of $\mathrm{SiN}$ was deposited onto silicon using a low-pressure chemical vapor deposition (LPCVD) method. $10 \mathrm{~nm}$ of $\mathrm{SiO}_{2}$ was formed by thermal oxidation at $800^{\circ} \mathrm{C}$. The substrates were primed with hexamethyldisilazane (HMDS) and then coated with TDUR-P3344 chemically amplified (CA) positive-tone resist (Tokyo Ohka Kogyo Co., Ltd.) and TARC AZ ${ }^{\circledR}$ Aquatar ${ }^{\circledR}$-VIII (Merck KGaA). The film thicknesses of the resist and TARC were $385 \mathrm{~nm}$ and $43 \mathrm{~nm}$, respectively. Exposure was performed using an FPA6000ES6a deep-UV scanner (Canon Inc.) equipped with a $\mathrm{KrF}(»=248 \mathrm{~nm})$ excimer laser light source. A CLEAN TRACK LITHIUS unit (Tokyo Electron Limited) was used as the coater/developer. A linear-drive (LD) nozzle was used to form a puddle of developer as it passed over the resist surface to be developed. Development was performed in a $0.26-\mathrm{N}$ aqueous solution of tetramethylammonium hydroxide (TMAH), and rinsing was performed using DI water.

\subsection{Analysis method}

To examine the number of cone defects that had been generated, the wafers were inspected using a KLA 2800 defect inspection tool (KLA-Tencor Corporation). X-ray photoelectron spectroscopy (XPS) analyses were performed to confirm the generation of resist residue after development. A Theta300XTR (Thermo Fisher Scientific Inc.) XPS tool was used for these analyses. To investigate the cause of cone defect generation, the metallic impurities that had accumulated on the wafer were analyzed. These metallic impurities were collected from the wafer surface by dissolving in a mixture of hydrofluoric acid (HF) and hydrogen peroxide $\left(\mathrm{H}_{2} \mathrm{O}_{2}\right)$. Subsequently, an elemental analysis of the collected liquid was performed by means of inductively coupled plasma mass spectrometry (ICP-MS). To analyze the sample for which resist remained on the wafer, ICP-MS was performed after the carbon element had been removed by an ashing process. An ELEMENT2 unit (Thermo Fisher Scientific Inc.) ICP-MS was used for these analyses.

\section{Results and Discussion}

3.1. Process factors affecting number of generated cone defects

After SiN dry etching using resist patterns fabricated directly onto the $\mathrm{SiN}$ as a mask, numerous SiN-based cone defects were detected in the photoresist openings. The size of these defects was about $30 \mathrm{~nm}$, and each had a cone shape (Fig. 1). It was found that all of the defects were of basically the same shape and size.

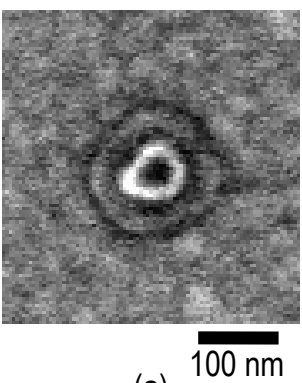

(a)

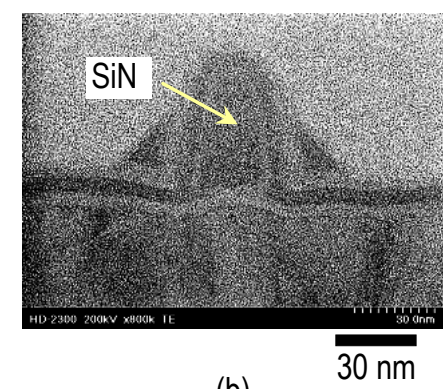

(b)
Figure 1. SEM image of cone defect after expanding due to film deposition: (a) Top view, (b) Cross-section.

As a first step, an investigation of the process factors that could influence the number of generated cone defects was performed. It was found that, when the lithography process was performed, the number of cone defects increased (Fig. 2). The unshared electron pair of the nitrogen atom in the SiN takes a negative charge and exhibits basicity. Because of that, when the resist pattern is formed on the $\mathrm{SiN}$, an acid deactivation reaction occurs at the interface between the resist and the $\mathrm{SiN}$ [1-4]. In the event of acid deactivation, there is the possibility of the resist polymer not dissolving in the developer. The resist patterns were fabricated on both $\mathrm{SiN}$ and $\mathrm{SiO}_{2}$ surfaces. These were then measured by XPS. 
(a)

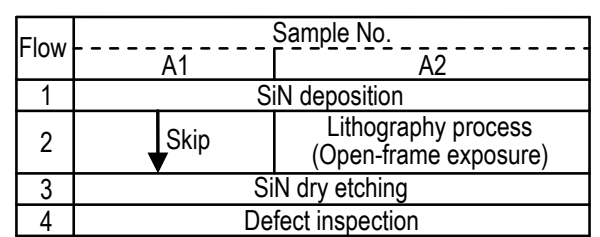

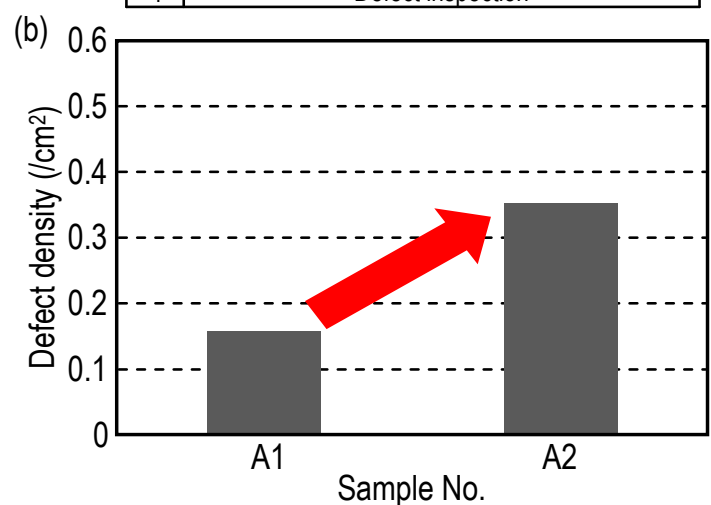

Figure 2. Change in number of cone defects as a result of executing lithography process: (a) Sample manufacturing flow, (b) Result of defect inspection.

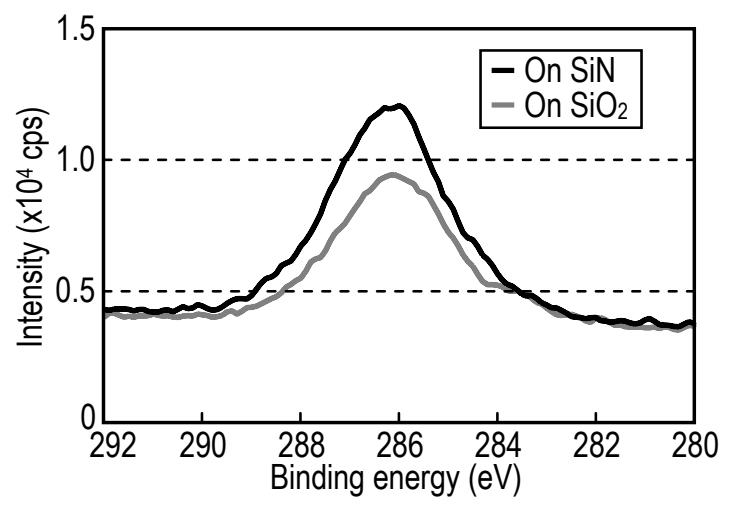

Figure 3. C1s XPS spectra of sample after lithography process has been performed on $\mathrm{SiN}$ and $\mathrm{SiO}_{2}$.

Figure 3 shows the XPS C1s peak for each of the measured samples. It was observed that the peak carbon intensity for those samples for which the resist pattern is fabricated on $\mathrm{SiN}$ is greater than that of the samples formed on $\mathrm{SiO}_{2}$. This implies that a resist residue was generated in the photoresist openings of the SiN samples. An assumption was made as to the possibility of the resist residue that was formed on the SiN acting as an etching mask which, in effect, led to the generation of the cone defects. However, numerous variations in the size, height, and shape of the resist residue were commonly observed. This contradicts the results obtained after SiN dry etching, when all of the cone defects were found to be of approximately the same size and shape. This led to doubts about the assumption that these cone defects are pattern-transferred from the resist residues.

In addition to during the lithographic process, the number of generated cone defects was also found to change during the wet process. Figure 4 shows the results of evaluating the variations in the detected cone defect density when using different types of wet processes. From these results, it was observed that the number of cone defects increased for sample B3 after hot phosphoric acid cleaning. On the other hand, the number of cone defects decreased in sample B4 which was processed with APM/HPM cleaning after hot phosphoric acid cleaning.
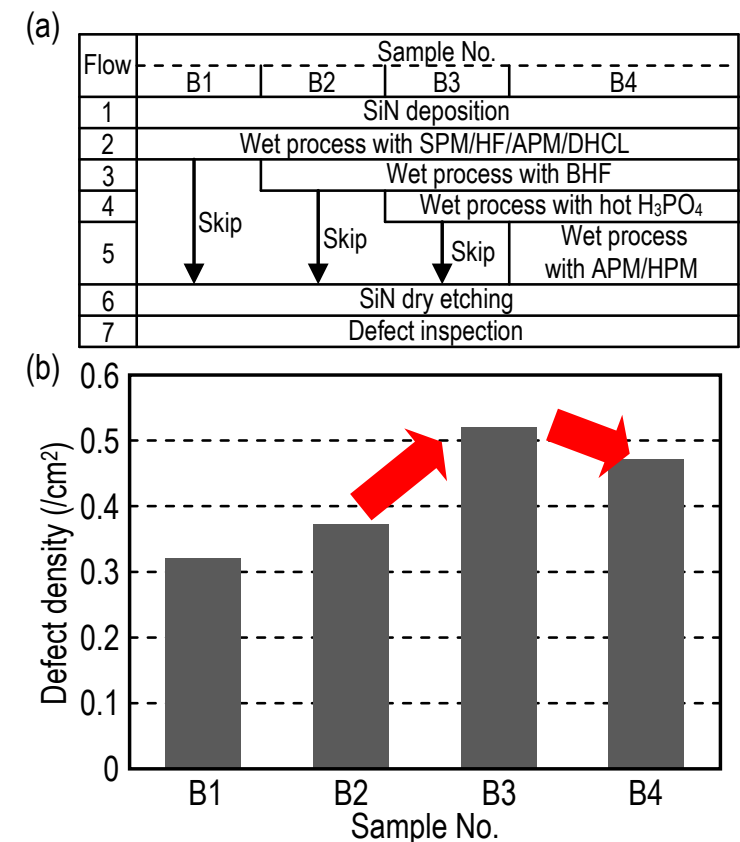

Figure 4. Change in number of cone defects as a result of executing each wet process: (a) Sample manufacturing flow, (b) Result of defect inspection.

For the hot phosphoric acid cleaning process, a NISON ${ }^{\circledR}$ VESPER system (Apprecia Technology Inc.), which enables effective etch selectivity control, in addition to phosphoric acid recycling, was utilized. The use of this system reduces the tool down-time and minimizes the chemical consumption. However, a disadvantage of such a system is the possible increase in the amount of metallic impurities resulting from the utilization of recycled phosphoric acid. It was assumed that cone defect generation 
increased during the hot phosphoric acid cleaning process because metallic impurities in the chemicals had adhered to the substrate. Regarding the reduction in the number of cone defects when APM/HPM cleaning was performed, it was assumed that the metal impurities that had been deposited during the hot phosphoric acid cleaning was effectively removed by the subsequent process. To verify this speculation, ICP-MS analyses were performed. Figure 5 shows the ICP-MS analyses carried out on the samples; $\mathrm{C} 1$ (unprocessed $\mathrm{SiN}$ ), $\mathrm{C} 2$ (SiN after hot phosphoric acid cleaning), C3 (SiN after hot phosphoric acid cleaning and APM/HPM cleaning), and $\mathrm{C} 4$ (SiN after hot phosphoric acid cleaning and APM/HPM cleaning, followed by a lithographic process consisting of open-frame exposures).

(a)

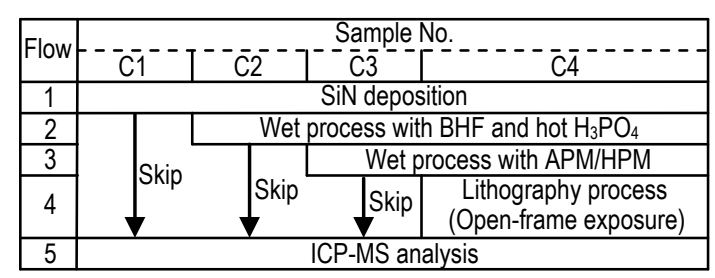

(b)

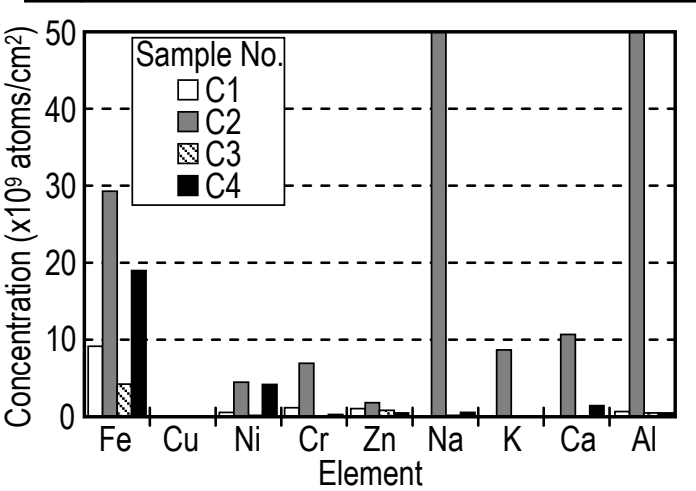

Figure 5. Change in amount of metal impurities on wafer as a result of executing wet and lithography processes: (a) Sample manufacturing flow, (b) Result of ICP-MS analysis.

As expected, numerous metallic impurities were detected in sample $\mathrm{C} 2$. These numbers translate into the high probability of metallic impurities influencing the generation of cone defects. Specifically, focusing on iron atoms, it was observed that the amount of this metal increased after hot phosphoric acid cleaning, decreased after APM/HPM cleaning, but also increased after the lithography process. These tendencies in the iron atoms were directly proportional to the increase and decrease in the number of cone defects. This naturally leads to the assumption that iron atoms in the metallic impurities greatly influence the generation of cone defects. As can be seen in Fig. 5, relatively abundant iron atoms were detected for sample $\mathrm{C} 4$ which had been subjected to lithography processing. Thus, an investigation to determine the source of the iron atoms in the lithography processes was undertaken.

\subsection{Iron atoms in lithography process}

During the lithography process, the wafer surface comes into contact with hexamethyldisilazane (HMDS), a thinner solvent (used for reducing resist consumption (RRC)), resist, top anti-reflection coating (TARC), developer, and DI water. For these materials, an investigation to determine the source of the iron atoms deposited on the sample surface was undertaken. Initially, an assumption was made that the iron atoms in the chemicals used for the HMDS, thinner, resist, and TARC were the main sources. Because metallic impurities had not been clearly detected on the $\mathrm{SiO}_{2}$ (Sample D1), the substrate used for the $\mathrm{SiO}_{2}$ was used for this evaluation.

(a)

\begin{tabular}{|c|c|c|}
\hline \multirow{2}{*}{ Flow } & \multicolumn{2}{|c|}{ Sample No. } \\
\hline & $\overline{\mathrm{D}} 2{ }^{-1-}$ & $\bar{D} \overline{3}$ \\
\hline 1 & \multicolumn{2}{|c|}{$\mathrm{SiO}_{2}$ deposition } \\
\hline 2 & \multicolumn{2}{|c|}{ Photoresist coating } \\
\hline 3 & TSkip & TARC coating \\
\hline 4 & \multicolumn{2}{|c|}{ Ashing } \\
\hline 5 & \multicolumn{2}{|c|}{ ICP-MS analysis } \\
\hline
\end{tabular}

(b)

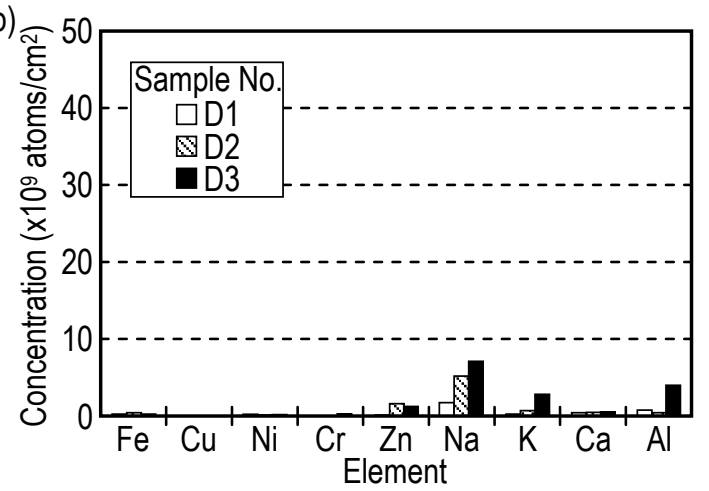

Figure 6. Change in amount of metal impurities on wafer after photoresist and TARC coating: (a) Sample manufacturing flow, (b) Result of ICP-MS analysis.

Figure 6 shows the metallic impurity analyses carried out for samples; D2 (prepared with HMDS priming and resist coating with RRC) and D3 (prepared with the 
same materials as D2 but with the addition of TARC). As these results showed, contrary to the initial assumption, only a small amount of metallic impurities were detected in both samples. Next, as shown in Fig. 7, ICP-MS analyses were carried out for the developer and DI water. Negligible amounts of impurity were found in the DI water, but 9 wt ppt of iron was detected in the developer solution.

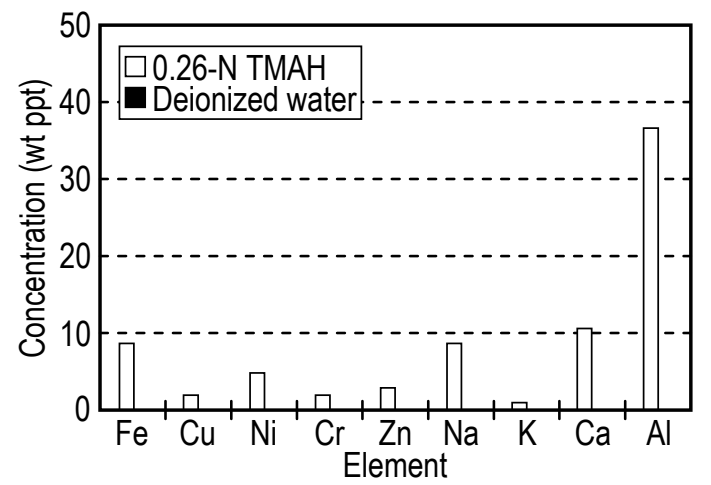

Figure 7. The amount of metal impurities contained in 0.26-N TMAH and deionized water.

This, however, is a relatively small value. Nevertheless, if all of the iron atoms in the developer were to be deposited on the wafer, it was calculated that the amount of iron detected on the wafer would be $15.4 \times 10^{9}$ atoms $/ \mathrm{cm}^{2}$ (with $168 \mathrm{~g} /$ wafer of developer discharged by the LD nozzle per development process, with $2 / 3$ of the developer solution assumed to fall on the wafer surface). The calculated amount of iron impurity deposited on the wafer surface is roughly the same as the amount of iron detected from sample C4, the sample subjected to the lithography process, as discussed in the previous section. This shows that the previously assumed small amount of 9 wt ppt iron impurities contained in the developer actually has a major impact on the number of cone defects that are generated. However, the amount of iron detected in the $\mathrm{SiO}_{2}$-based samples E1 (lithographic process without exposure) and E2 (lithographic process with exposure), both of which were subjected to the lithography process, was smaller than the above-mentioned calculation value (Fig. 8).
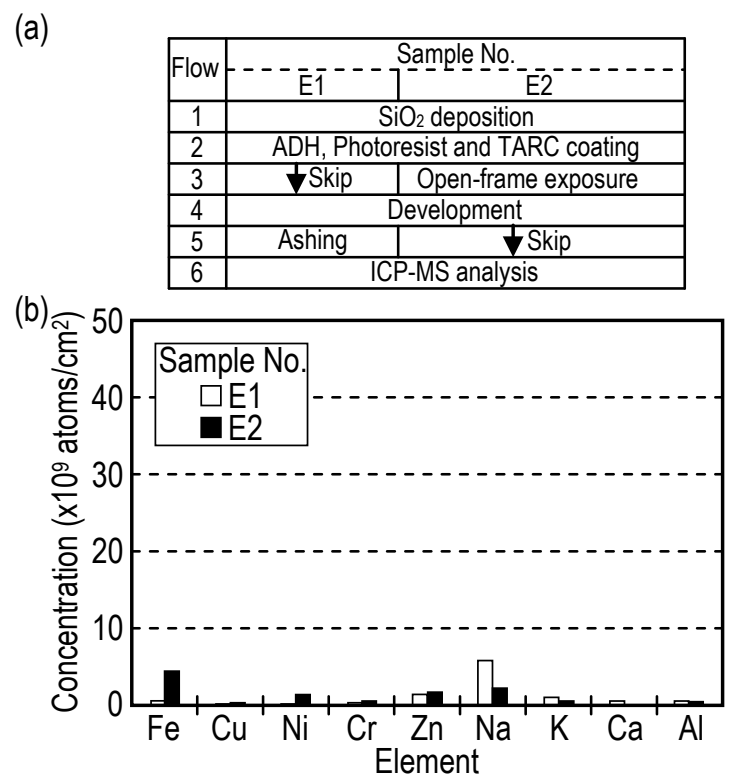

Figure 8. Change in amounts of metal impurities on wafer after development: (a) Sample manufacturing flow, (b) Result of ICP-MS analysis.

The assumption was that some of the metallic impurities were discharged outside the wafer (drained out) with the developer. The amount of iron impurities detected on the surface of E2 was smaller than that found on C4. The main difference between these samples is the under-layer material, with $\mathrm{SiO}_{2}$ being used for $\mathrm{E} 2$ and $\mathrm{SiN}$ used for $\mathrm{C} 4$. The increase in the amount of iron impurities for sample $\mathrm{C} 4$ was assumed to be due to the $\mathrm{SiN}$ surface, which easily traps these iron impurities.

(a)

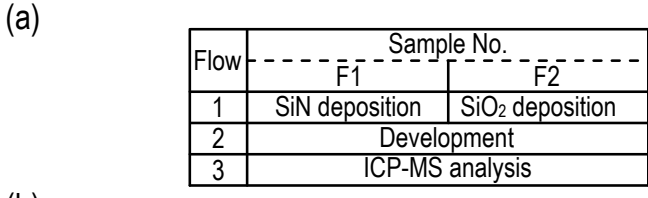

(b)

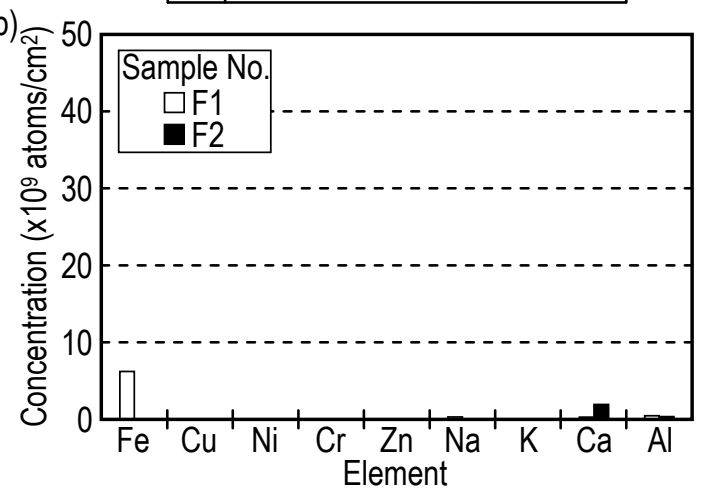

Figure 9. Change in amount of metal impurities on $\mathrm{SiN}$ and $\mathrm{SiO}_{2}$ after development: (a) Sample manufacturing flow, (b) Result of ICP-MS analysis. 
Figure 9 shows the ICP-MS analysis results for developed samples F1 and F2, with $\mathrm{SiN}$ and $\mathrm{SiO}_{2}$ under-layers, respectively. Based on these results, it was confirmed that $\mathrm{SiN}$ is more prone to trap the iron impurities in the developer, relative to the $\mathrm{SiO}_{2}$. Given these results, it was assumed that combining (adding) the amount of detected iron impurity for samples E2 (analysis results for exposed resist on $\mathrm{SiO}_{2}$ ) and $\mathrm{F} 1$ (analysis results for only SiN surface after contact with developer) should result in values similar to those obtained with sample C4 (analysis of exposed resist on $\mathrm{SiN}$ ). However, it is interesting that these combined values were only half of those obtained with sample C4.

A proposed mechanism as to why the abundance of iron impurities was detected in sample $\mathrm{C} 4$ is shown in Fig. 10. As discussed in Fig. 3, when the resist patterns are fabricated on SiN, resist residue is generated in the resist openings. This resist residue consists of resist polymers with numerous polar groups for which the deprotective reaction partially progresses but for which total dissolution in the developer is not achieved. As polymers with a high concentration of polar groups are highly compatible with the developer, the resist residue swells upon contact with the developer solution. The inter-polymer gap increases due to this swelling phenomenon, allowing the developer solution to permeate the spaces between the polymers. Moreover, due to these extended inter-polymer gaps, the polymer's orientation direction can very easily vary. In such an environment, contact between a resist polymer with numerous polar groups in the resist residue and iron impurities in the developer is assumed to occur easily such that it forms a cluster complex as a result of hydrophobic interaction as the driving force [5-7]. This hypothesis is viewed as being the reason why an abundance of iron impurities was detected in sample $\mathrm{C} 4$.

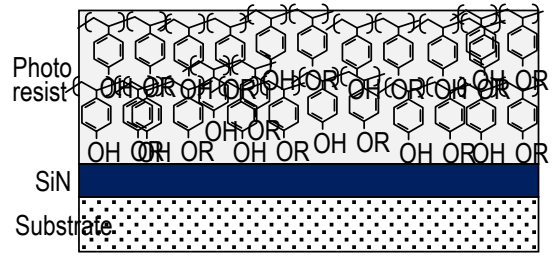

(a) Photoresist coating

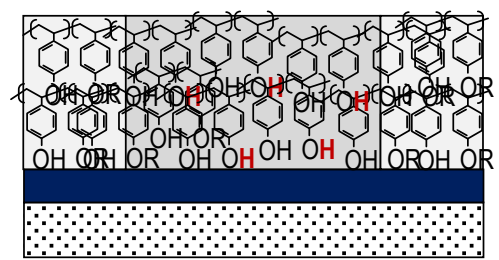

(d) Deprotection reaction

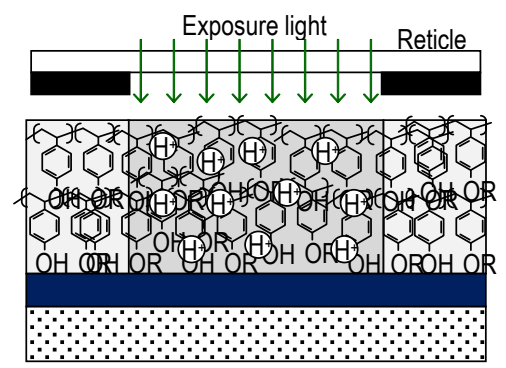

(b) Exposure

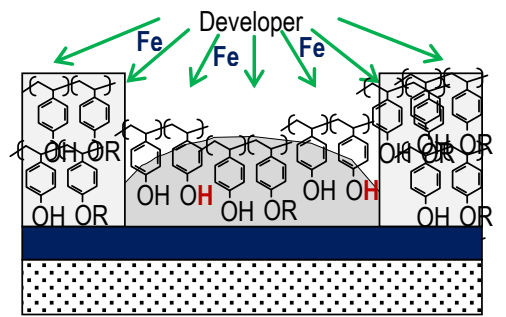

(e) Resist residue generation after development

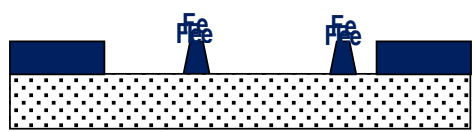

(g) Cone defect generation after SiN etching

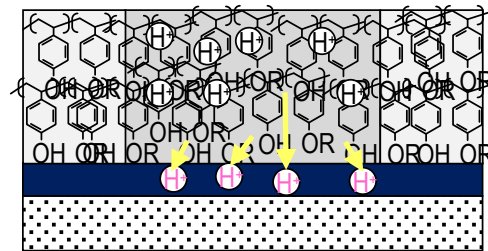

(c) Acid deactivation between resist and SiN interface

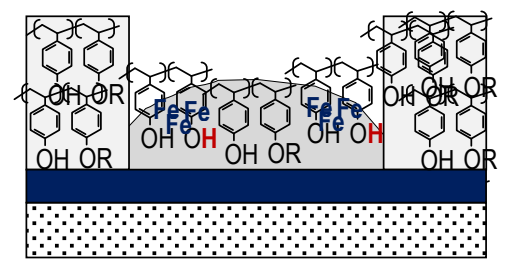

(f) Complexation by deproteced polymer and iron atoms

Figure 10. Cone defect generation model 
To prove this hypothesis, another sample, G1, was prepared on $\mathrm{SiO}_{2}$. Sample G1 was open-frame exposed with exposure doses low enough to prevent complete dissolution of the resist in the developer solution. This sample produced a state in which resist residue is generated on $\mathrm{SiO}_{2}$ even though the resist residue is usually not generated on $\mathrm{SiO}_{2}$. If this hypothesis is correct, these conditions should induce the significant metal contamination as detected in sample $\mathrm{C} 4$. As a result, an abundance of iron atoms was detected in sample G1 (Fig. 11). The results obtained through these investigations show that one of the main factors in the generation of cone defects was the fact that the resist residue on $\mathrm{SiN}$ had trapped the metallic impurities in the developer (especially, iron), forming a cluster complex between the polymer and metal which then acts as an etching mask in the subsequent process.

(a)

\begin{tabular}{|c|c|}
\hline Flow & Sample No. \\
\hline 1 & $\mathrm{SiO}_{2}$ deposition \\
\hline 2 & $\mathrm{ADH}$, Photoresist and TARC coating \\
\hline 3 & Open frame exposure with a low-exposure doses \\
\hline 4 & Development \\
\hline 5 & ICP-MS analysis \\
\hline
\end{tabular}

(b)

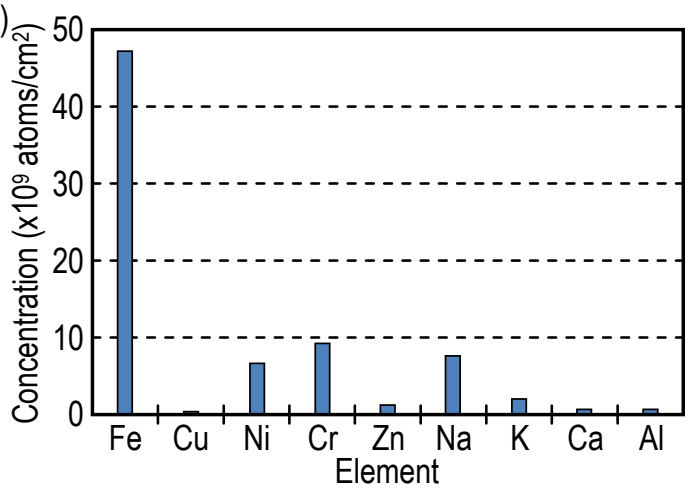

Figure 11. Change in amount of metal impurities on wafer with resist residue: (a) Sample manufacturing flow, (b) Result of ICP-MS analysis.

\subsection{Metal-trapping properties of $\mathrm{SiN}$}

As discussed earlier, the SiN surface trapped more of the iron impurities in the developer than the $\mathrm{SiO}_{2}$ surface. Hot phosphoric acid cleaning for both samples was performed using the VESPER system ( 9 $\mathrm{nm}$ of SiN was removed). After this process, an abundance of metallic impurities was still found adhering to the SiN (Sample C2, shown in Fig. 5). In contrast, for the $\mathrm{SiO}_{2}$ sample, the amount of metallic impurities was found to have decreased (Sample H1, shown in Fig. 12). When the same process was performed until the entire SiN layer was removed, the presence of metallic impurities was shown to decrease (Sample H2 in Fig. 12). These results show that these metallic impurities are easily trapped in the SiN surface. Kobayashi, et al. reported that the formation energy for the metallic atoms on the SiN surface is lower than that when the same atoms are present in the SiN (inside the film layer) [8, 9]. It was thus speculated that the trap sites, where these atoms easily settle, are present in the SiN surface. Metallic impurities concentrate and stabilize in these SiN trap sites without diffusing into the SiN film. When this occurs, the metallic impurities accumulated on these trap sites act as an etching mask in the subsequent process, resulting in the formation of cone defects.

(a)

\begin{tabular}{|c|c|c|}
\hline Flow & $\ldots \ldots$ & Sample No. $\ldots$ \\
\hline 1 & \multicolumn{2}{|c|}{$\mathrm{SiO}_{2}$ deposition } \\
\hline 2 & $\downarrow$ Skip & SiN deposition \\
\hline 3 & $\begin{array}{c}\text { Wet process with } \mathrm{BHF} \\
\text { and hot } \mathrm{H}_{3} \mathrm{PO}_{4}\end{array}$ & $\begin{array}{c}\text { Wet process with BHF } \\
\text { and hot } \mathrm{H}_{3} \mathrm{PO}_{4} \\
\text { (remove all SiN) }\end{array}$ \\
\hline 4 & \multicolumn{2}{|c|}{ ICP-MS analysis } \\
\hline
\end{tabular}

(b)

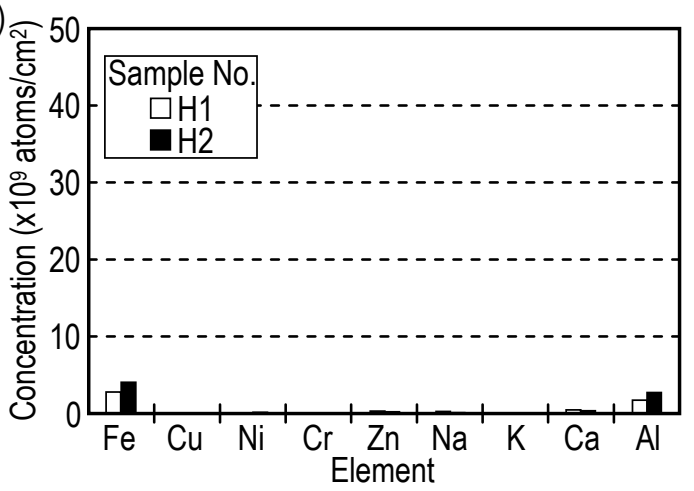

Figure 12. Change in amount of metal impurities on wafer after hot $\mathrm{H}_{3} \mathrm{PO}_{4}$ wet process on $\mathrm{SiO}_{2}$ : (a) Sample manufacturing flow, (b) Result of ICP-MS analysis.

3.4. Measures to reduce the number of cone defects

On the basis of the evaluation results obtained so far, three measures to reduce the number of cone defects are proposed.

(1) Utilize resists with a high development contrast to suppress the generation of resist 
residues that contain polymer with numerous polar groups which, in effect, trap metal impurities. (2) Reduce the amount of metallic impurities in the developer and possibly the DI water that comes into contact with the resist residue. Moreover, on the basis of the ICP-MS analysis results for Samples C4 and E2, there is a need to minimize the amount of iron contained in the developer to $<2.1 \mathrm{wt} p \mathrm{pt}$ to reduce the amount of iron trapped by the $\mathrm{SiN}$ to the same level as those obtained with $\mathrm{SiO}_{2}$. Thus, another measure is to (3) reduce the amount of metallic impurities contained in the chemicals that come into contact with the SiN surface (HMDS, resist, TARC, developer, DI water, or chemical solutions used for wet processing).

\section{Conclusion}

Based on investigations into the cone defects detected after the dry etching of SiN, the following factors were found to be the main causes; (1) the resist residue generated on the $\mathrm{SiN}$ trapped the metallic impurities (especially, iron) present in the developer, thus forming a polymer-metal impurity complex and (2) in the SiN surface, there are trap sites in which metallic atoms accumulate.

To reduce the number of cone defects, suppression of resist residue generation through the application of resists with a high development contrast will be necessary. Another measure would be the reduction of the amount of metallic impurities in the developer solution. Lastly, the minimization of metallic impurities in the chemicals discharged onto the SiN surface is also an effective measure.

Moreover, through these evaluations, it was found that the iron atoms in the developer greatly influence cone defect generation. It is thus necessary to reduce the amount of iron impurities in the developer solution to a maximum of $2.1 \mathrm{wt} \mathrm{ppt}$ to achieve low number of defects at the same level as those obtained for $\mathrm{SiO}_{2}$. The authors are aware that these measures are not easily implementable. However, such measures will become indispensable for improving the manufacturing yield of semiconductor devices from the viewpoint of reducing the number of defects.

\section{References}

1. G. Breyta, D. C. Hofer, and H. Ito, $J$. Photopolym. Sci. Technol., 7 (1994), 433.

2. A. Usujima, K. Tago, A. Oikawa, and K. Nakagawa, Proc. SPIE, 2438 (1995), 529.

3. R. Yamanaka, T. Hattori, T. Mine, and T. Tanaka, Japanese Journal of Applied Physics, 34 (1995), 6794.

4. T. Fukushima, N. Morosawa, Y. Matsuura, and S. Mori, J. Photopolym. Sci. Technol., 10 (1997), 457.

5. Y. Mori, H. Yokoi, and Y. Fujise, Polymer Journal, 27 (1995), 271.

6. C. Yang, F. Ko, M. Wang, T. Wang, and T. Huang, Proc. SPIE, 3998 (2000), 589.

7. T. Kohyama, Proc. SPIE, 8325 (2012), 83252 I- 1.

8. S. Kobayashi, D. Shibata, K. Sueoka, J. Komachi, and K. Saga, 61th Spring Meeting of The Japan Society of Applied Physics (2014), 13-027.

9. D. Shibata, S. Kobayasih, K. Sueoka, J. Komachi, and K. Saga, ECS Trans., 64 (2014), 229. 